Shanguang Yang, Jingjing Liu, Zhudan Jin, Wei Tian, Hao Sun and Mingliang Wang*

\title{
A novel one-pot approach to oxidative aromatization and bromination of pyrazolidin-3-one with $\mathrm{HBr}-\mathrm{H}_{2} \mathrm{O}_{2}$ system
}

https://doi.org/10.1515/hc-2018-0046

Received March 19, 2018; accepted March 27, 2018; previously published online May 7, 2018

Abstract: An efficient and green one-pot method for the oxidative aromatization and bromination of pyrazolidin3-ones under mild conditions with a $\mathrm{HBr}-\mathrm{H}_{2} \mathrm{O}_{2}$ system was developed. A mechanism was proposed.

Keywords: aromatization; bromination; bromopyrazol; hydrogen bromide; hydrogen peroxide; one-pot; oxidative; pyrazolidin-3-one.

\section{Introduction}

Pyrazole derivatives attract attention due to their excellent pharmacological properties [1-4]. Halogenated pyrazolols [5, 6], especially 4-bromo derivatives, are precursors for the synthesis of functionalized pyrazoles [7-9], such as pharmaceuticals [10], multifunctional materials [11, 12], fused- [13] and spiro-heterocyclic compounds [14]. Various protocols for the synthesis of brominated pyrazolols have been reported. However, bromination of pyrazolols with $\mathrm{Br}_{2}$ in acetic acid is carried out under harsh conditions and requires careful manipulation [15]. Efforts have been made by Ahmed et al. [16] to use the photolysis of $N$-bromosuccinimide as the source of bromine [16]. Due to the formation of the coupling by-products, bromopyrazolols are afforded in low yields. Although $N$-bromobenzamide [17] and dibromoisocyanuric acid [18] have also been explored as mild brominating agents for pyrazolols, these approaches have met with limited success.

Recently, pyrazolidinones have been reported as substrates for the synthesis of pyrazolols [7, 11, 12, 19-23]. Unfortunately, few efficient methods for direct access

*Corresponding author: Mingliang Wang, School of Chemistry and Chemical Engineering, Southeast University, Nanjing 211189, P. R. China, e-mail: wangmlchem@seu.edu.cn. http://orcid. org/0000-0002-3934-6100

Shanguang Yang, Jingjing Liu, Zhudan Jin, Wei Tian and Hao Sun: School of Chemistry and Chemical Engineering, Southeast University, Nanjing 211189, P. R. China to 4-bromopyrazolol from pyrazolidinone have been reported. Traditionally, there are two steps in the synthesis of 4-bromopyrazolol from pyrazolidinone [7, 11, 12] that involve aromatization of pyrazolidin-3-ones to give pyrazol-3-ols and bromination of pyrazol-3-ols [7]. This protocol requires the use of a transition-metal catalyst, extremely toxic liquid bromine, a long reaction time, multi-step manipulations and the final yield is only moderate. In recent years, brominations with safe bromination reagents and green co-oxidants have received growing attention because of their greenness and high efficiency [24]. Herein, we report a novel one-pot, metal-free, atomeconomic and highly effective method for the preparation of 4-bromopyrazol-3-ol from pyrazolidin-3-one using a $\mathrm{HBr}-\mathrm{H}_{2} \mathrm{O}_{2}$ system under mild conditions.

\section{Results and discussion}

The starting compounds 1 (Scheme 1) were commercially available or easily prepared according to previously published procedures [7, 11, 12, 19-23]. Initially, 1-(4-chlorophenyl)-pyrazolidin-3-one (1a) was treated with various amounts of hydrogen peroxide and hydrobromic acid using various solvents. It was found that the use of different solvents including carbon tetrachloride, chloroform, dichloromethane, methanol and $N, N$-dimethylformamide had little effect on the outcome, affording the yield of the product $\mathbf{2 a}$ in the range from $72 \%$ to $90 \%$. Nevertheless, the highest yield of $90 \%$ was obtained for the reaction conducted in chloroform. Under optimized conditions, the synthesis of $2 \mathrm{a}$ was conducted in chloroform at $60^{\circ} \mathrm{C}$ using 3 equivalents of $\mathrm{H}_{2} \mathrm{O}_{2}$ and 1 equivalent of $\mathrm{HBr}$.

Decreasing the amount of hydrogen peroxide to 1 equivalent or 2 equivalents resulted in a decrease of the yield of the product $\mathbf{2 a}$. On the other hand, an increase in the amount of hydrogen peroxide from 3 equivalents to 4 equivalents did not affect the yield. With the optimized reaction conditions for $\mathbf{2 a}$, a wide range of 4-bromo-3-hydroxypyrazolidines were synthesized (Scheme 1). The reaction proceeds well with pyrazolidin-3-ones containing a phenyl group, a substituted phenyl group or a pyridinyl 


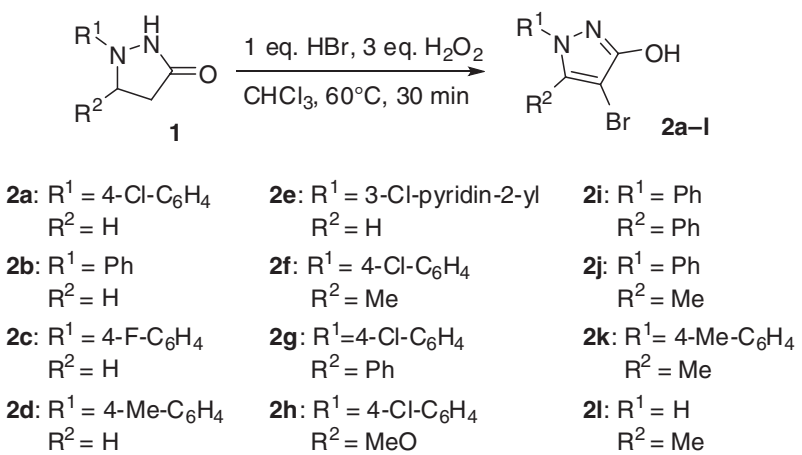

Scheme 1

group. With an increase in the electron-donating capability of the substituent at the 5-position of pyrazolidin3-ones $\left(\mathrm{MeO}>\mathrm{CH}_{3}>\mathrm{H}\right)$, the efficiency of the reaction is increased in the same order.

Interestingly, when the reaction with 5 equivalents of $\mathrm{HBr}$ was carried out for $3 \mathrm{~h}$ (Scheme 2), the corresponding dibrominated products $\mathbf{3} \mathbf{b}, \mathbf{j}$ were obtained accompanied by the corresponding monobrominated products $\mathbf{2} \mathbf{b}, \mathbf{j}$ in moderate yields. However, in the case of compound $\mathbf{1 i}$ only monobrominated product $\mathbf{2 i}$ was acquired in high yield. For further confirmation of the structure of the series, a single crystal of $\mathbf{3} \mathbf{j}$ was obtained and subjected to $\mathrm{X}$-ray diffraction analysis (Figure 1).

To gain access to the mechanism, the experiments were carried out as shown in Scheme 3. When the

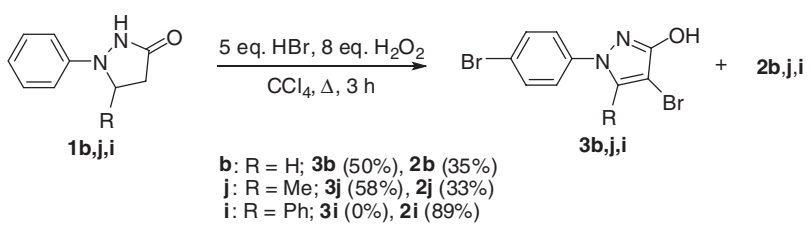

Scheme 2

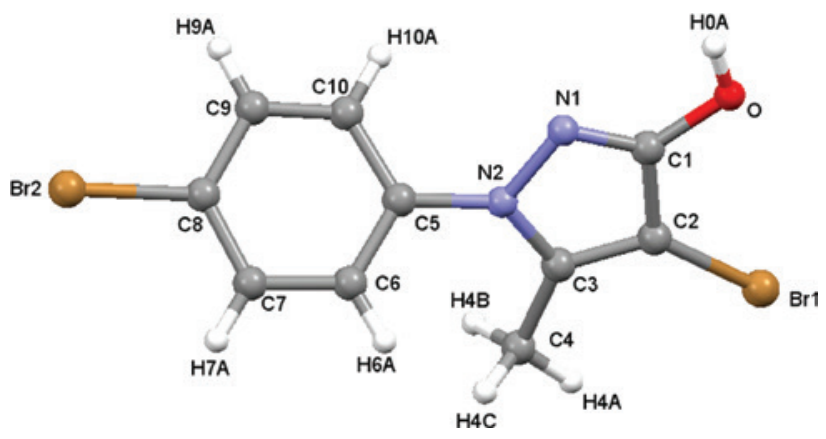

Figure 1 Molecular structure of $\mathbf{3} \mathbf{j}$ with atom labeling.

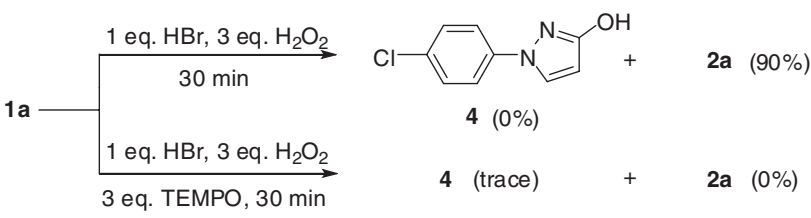

Scheme 3

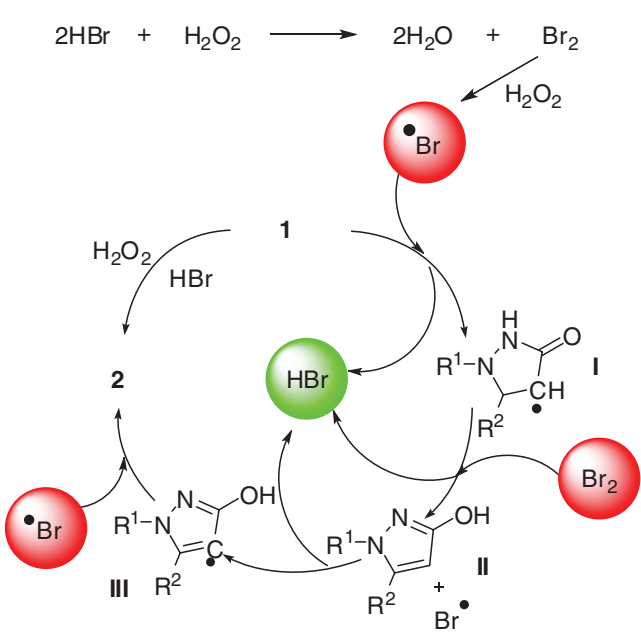

Scheme 4

reaction under optimized conditions was performed in the presence of 2,2,6,6-tetramethylpiperidine-1-oxyl (TEMPO) as a radical scavenger, only a trace amount of 1-(4-chlorophenyl)-1H-pyrazol-3-ol (4) was detected, suggesting a radical pathway. This finding and previous reports [25-27], are consistent with the mechanism suggested in Scheme 4. First, compound 1 undergoes a reaction with a bromine radical to generate the corresponding radical intermediate $\mathbf{I}$, which is subsequently converted to the substituted pyrazol-3-ol II by oxidation with molecular bromine. Then, in the presence of the bromine radical, the corresponding radical species III is generated from the intermediate compound II. Finally, the reaction of the intermediate product III with the bromide radical furnishes the observed 4-bromopyrazol-3-ol 2.

\section{Conclusions}

A green and efficient protocol to prepare substituted 4-bromo-pyrazol-3-ols 2 from pyrazolidin-3-ones 1 under mild conditions in excellent yields was described. The corresponding dibromination products $\mathbf{3} \mathbf{b}, \mathbf{j}$ were also obtained in moderate yields under harsh conditions. 


\section{Experimental}

Proton nuclear magnetic resonance ( ${ }^{1} \mathrm{H}$ NMR) spectra were obtained on a Bruker spectrometer operating at $600 \mathrm{MHz}$ with dimethyl sulfoxide- $d_{6}$ as solvent and tetramethylsilane as internal standard. Infrared (IR) spectra were obtained using $\mathrm{KBr}$ disks on a Bomem Michelson Series Fourier-transform infrared spectrometer. The mass spectrometry (MS) data were recorded with an Agilent 7890A-5975C instrument. Melting points were obtained on an X-4 microscope electrothermal apparatus (Taike, China) and are uncorrected. Elemental analyses were carried out on a Vario EL III analyzer. Spectral data for compounds $\mathbf{2} \mathbf{b}, \mathbf{3 b}$ and $\mathbf{4}$ are virtually identical with the reported values $[9,20,28]$.

\section{Synthesis of 4-bromopyrazol-3-ones $2 \mathrm{a}-\mathrm{l}$}

A mixture of 1a-l $(0.01 \mathrm{~mol})$ in $\mathrm{CHCl}_{3}(10 \mathrm{~mL})$ and $40 \%$ hydrobromic acid $(40 \%, 0.01 \mathrm{~mol})$ was stirred at $60^{\circ} \mathrm{C}$ and treated dropwise for 10 min with an aqueous solution of $\mathrm{H}_{2} \mathrm{O}_{2}(30 \%, 0.03 \mathrm{~mol})$, and stirring was continued for an additional $20 \mathrm{~min}$. The resultant precipitate of 2a-1 was filtered off and crystallized from ethanol. The filtrate was concentrated and the residue was subjected to silica gel chromatography eluting with ethyl acetate/petroleum ether (10:1) to give an additional amount of $\mathbf{2 a - 1}$.

4-Bromo-1-(4-chlorophenyl)-1H-pyrazole-3-ol (2a) White solid; mp 192-194 ${ }^{\circ} \mathrm{C}$; yield 90\%; ${ }^{1} \mathrm{H}$ NMR: $\delta 11.13$ (s, $\left.1 \mathrm{H}, \mathrm{OH}\right), 8.58(\mathrm{~s}, 1 \mathrm{H}, \mathrm{CH})$, 7.71 (d, $J=9$ Hz, 2H, Ar), 7.51 (d, J=9 Hz, 2H, Ar); IR: 3440, 2971, 1617, 1563, 1494, 1392, 1306, $1105 \mathrm{~cm}^{-1}$; MS: $\mathrm{m} / z 274.3\left[(\mathrm{M}+1)^{+}, 100 \%\right]$. Anal. Calcd for $\mathrm{C}_{9} \mathrm{H}_{6} \mathrm{BrClN}_{2} \mathrm{O}$ : C, 39.52; H, 2.21; N, 10.24. Found: C, 39.81; H, 2.03; N, 10.02 .

4-Bromo-1-phenyl-1H-pyrazole-3-ol (2b) Yellow solid; mp 188$190^{\circ} \mathrm{C}$; yield 91\%; ${ }^{1} \mathrm{H}$ NMR: $\delta 11.02(\mathrm{~s}, 1 \mathrm{H}, \mathrm{OH}), 8.54(\mathrm{~s}, 1 \mathrm{H}, \mathrm{CH}), 7.69(\mathrm{~d}$, $J=8 \mathrm{~Hz}, 2 \mathrm{H}, \mathrm{Ar}$ ), 7.45 (t, $J=8 \mathrm{~Hz}, 2 \mathrm{H}, \mathrm{Ar}), 7.23$ (t, $J=8 \mathrm{~Hz}, 1 \mathrm{H}, \mathrm{Ar}) ; \mathrm{IR}: 3449$, 2927, 1620, 1550, 1493, 1309, $1102 \mathrm{~cm}^{-1}$; MS: $\mathrm{m} / z 240.1$ [(M+1)+, 100\%].

4-Bromo-1-(4-fluorophenyl)-1H-pyrazole-3-ol (2c) White solid; mp 219-221 ${ }^{\circ} \mathrm{C}$; yield 89\%; ${ }^{1} \mathrm{H}$ NMR: $\delta 11.01$ (s, $\left.1 \mathrm{H}, \mathrm{OH}\right), 8.51$ (s, $1 \mathrm{H}, \mathrm{CH}$ ), 7.70 (m, 2H, Ar), 7.30 (t, J=9 Hz, 2H, Ar); IR: 3451, 2971, 1622, 1568, 1519, 1404, 1311, 1240, $1087 \mathrm{~cm}^{-1}$; MS: $\mathrm{m} / z 258.1$ [(M+1)+, 100\%]. Anal. Calcd for $\mathrm{C}_{9} \mathrm{H}_{6} \mathrm{BrFN}_{2} \mathrm{O}: \mathrm{C}, 42.05 ; \mathrm{H}, 2.35$; N, 10.90. Found: C, 42.22; H, 2.21; N, 10.68 .

4-Bromo-1-(4-methylphenyl)-1H-pyrazole-3-ol (2d) Yellow solid; mp 183-185 ${ }^{\circ}$; yield 92\%; ${ }^{1} \mathrm{H}$ NMR: $\delta 10.93(\mathrm{~s}, 1 \mathrm{H}, \mathrm{OH}), 8.47(\mathrm{~s}, 1 \mathrm{H}, \mathrm{CH})$, 7.56 (d, $J=8 \mathrm{~Hz}, 2 \mathrm{H}, \mathrm{Ar}$ ), 7.24 (d, $J=8 \mathrm{~Hz}, 2 \mathrm{H}, \mathrm{Ar}$ ), 2.31 (s, 3H, $\mathrm{CH}_{3}$ ); IR: 3454, 2964, 1625, 1548, 1381, 1327, $1082 \mathrm{~cm}^{-1}$; MS: $\mathrm{m} / z 254.2\left[(\mathrm{M}+1)^{+}\right.$, $100 \%]$. Anal. Calcd for $\mathrm{C}_{10} \mathrm{H}_{9} \mathrm{BrN}_{2} \mathrm{O}: \mathrm{C}, 47.46 ; \mathrm{H}, 3.58$; N, 11.07. Found: C, 47.31; H, 3.73; N, 10.88 .

4-Bromo-1-(3-chloropyridin-2-yl)-1H-pyrazole-3-ol (2e) Yellow solid; mp $233-235^{\circ} \mathrm{C}$; yield 91\%; ${ }^{1} \mathrm{H}$ NMR: $\delta 11.48(\mathrm{~s}, 1 \mathrm{H}, \mathrm{OH}), 8.49$ (s, 1H, CH), 7.98 (d, J=8 Hz, 1H, Ar), 7.60 (d, J=8 Hz, 1H, Ar), 7.37 (d, $J=8 \mathrm{~Hz}, 1 \mathrm{H}, \mathrm{Ar}$ ); IR: 3451, 2965, 1626, 1582, 1444, 1389, 1305, 1261, $1068 \mathrm{~cm}^{-1}$; MS: $\mathrm{m} / z 273.1\left[(\mathrm{M}+1)^{+}, 100 \%\right]$. Anal. Calcd for $\mathrm{C}_{8} \mathrm{H}_{5} \mathrm{BrClN}_{3} \mathrm{O}: \mathrm{C}, 35.00 ; \mathrm{H}, 1.84 ; \mathrm{N}, 15.31$. Found: C, 34.83; H, 2.02; $\mathrm{N}, 15.50$.
4-Bromo-5-methyl-1-(4-chlorophenyl)-1H-pyrazol-3-ol (2f) Yellow solid; mp $221-223^{\circ} \mathrm{C}$; yield 92\%; ${ }^{1} \mathrm{H}$ NMR: $\delta 10.80(\mathrm{~s}, 1 \mathrm{H}$, $\mathrm{OH}$ ), 7.55 (m, 2H, Ar), 7.51 (m, 2H, Ar), 2.28 (s, 3H, $\mathrm{CH}_{3}$ ); IR: 3455, 2978, 1617, 1545, 1510, 1393, $1102 \mathrm{~cm}^{-1}$; MS: $m / z$ 288.2 [(M+1)+, 81\%]. Anal. Calcd for $\mathrm{C}_{10} \mathrm{H}_{8} \mathrm{BrClN}_{2} \mathrm{O}: \mathrm{C}, 41.77 ; \mathrm{H}, 2.80$; N, 9.74. Found: C, 41.61; H, 2.93; N, 9.89.

4-Bromo-5-phenyl-1-(4-chlorophenyl)-1H-pyrazol-3-ol (2g) White solid; mp 253-255 ${ }^{\circ}$; yield 94\%; ${ }^{1} \mathrm{H}$ NMR: $\delta 11.07$ (s, $\left.1 \mathrm{H}, \mathrm{OH}\right), 7.43(\mathrm{~m}$, 3H, Ar), 7.39 (d, J=9 Hz, 2H, Ar), 7.30 (m, 2H, Ar), 7.21 (d, J=9 Hz, 2H, Ar); IR: 3462, 2973, 1552, 1496, 1331, $1075 \mathrm{~cm}^{-1}$; MS: $\mathrm{m} / z 350.3\left[(\mathrm{M}+1)^{+}\right.$, 100\%]. Anal. Calcd for $\mathrm{C}_{15} \mathrm{H}_{10} \mathrm{BrClN}_{2} \mathrm{O}: \mathrm{C}, 51.53 ; \mathrm{H}, 2.88 ; \mathrm{N}, 8.01$. Found: C, 51.34; H, 3.06; N, 8.22.

4-Bromo-5-methoxy-1-(4-chlorophenyl)-1H-pyrazol-3-ol (2h) Yellow solid; mp $228-230^{\circ} \mathrm{C}$; yield $95 \%$; ${ }^{1} \mathrm{H}$ NMR: $\delta 11.37(\mathrm{~s}, 1 \mathrm{H}$, $\mathrm{OH}$ ), 7.65 (m, 2H, Ar), 7.38 (m, 2H, Ar), 3.74 (s, 3H, OCH $)$ ) IR: 3452, 2961, 1632, 1560, 1503, 1402, 1314, 1207, $1054 \mathrm{~cm}^{-1}$; MS: $\mathrm{m} / z$ 300.4 $\left[(\mathrm{M}+1)^{+}, 100 \%\right]$. Anal. Calcd for $\mathrm{C}_{10} \mathrm{H}_{8} \mathrm{BrClN}_{2} \mathrm{O}_{2}: \mathrm{C}, 39.57 ; \mathrm{H}, 2.66$; N, 9.23. Found: C, 39.48; H, 2.79; N, 9.38.

4-Bromo-1,5-diphenyl-1H-pyrazol-3-ol (2i) Yellow solid; mp 242$244^{\circ} \mathrm{C}$; yield 93\%; ${ }^{1} \mathrm{H}$ NMR: $\delta 10.98$ (s, $\left.1 \mathrm{H}, \mathrm{OH}\right), 7.41$ (m, 3H, Ar), 7.29 (m, 5H, Ar), 7.14 (d, J=8 Hz, 2H, Ar); IR: 3453, 2965, 1624, 1541, 1444, 1335, 1252, $1085 \mathrm{~cm}^{-1}$; MS: $\mathrm{m} / z$ 314.1 [(M+1)+, 100\%]. Anal. Calcd for $\mathrm{C}_{15} \mathrm{H}_{11} \mathrm{BrN}_{2} \mathrm{O}: \mathrm{C}, 57.16 ; \mathrm{H}, 3.52 ; \mathrm{N}$, 8.89. Found: C, 57.37; H, 3.38; N, 8.71.

4-Bromo-5-methyl-1-phenyl-1H-pyrazol-3-ol (2j) White solid; mp 218-220 ${ }^{\circ}$; yield 91\%; ${ }^{1} \mathrm{H}$ NMR: $\delta 10.71(\mathrm{~s}, 1 \mathrm{H}, \mathrm{OH}), 7.49$ (m, 4H, Ar), 7.36 (t, $J=7 \mathrm{~Hz}, 1 \mathrm{H}, \mathrm{Ar}), 2.27$ (s, 3H, $\mathrm{CH}_{3}$ ); IR: 3450, 2969, 1623, 1549, 1496, 1409, 1328, 1270, $1103 \mathrm{~cm}^{-1}$; MS: $\mathrm{m} / z 254.1$ [(M+1)+, 100\%]. Anal. Calcd for $\mathrm{C}_{10} \mathrm{H}_{9} \mathrm{BrN}_{2} \mathrm{O}: \mathrm{C}, 47.46 ; \mathrm{H}, 3.58$; N, 11.07. Found: C, 47.63; H, 3.81; N, 10.86 .

4-Bromo-5-methyl-1-(4-methylphenyl)-1H-pyrazol-3-ol (2k) White solid; mp $232-234^{\circ} \mathrm{C}$; yield $92 \%$; ${ }^{1} \mathrm{H}$ NMR: $\delta 10.64$ (s, 1H, OH), 7.35-7.28 (m, 4H, Ar), 2.35 (s, 3H, $\mathrm{CH}_{3}$ ), 2.24 (s, 3H, $\mathrm{CH}_{3}$ ); IR: 3452, 2974, 1627, 1553, 1515, 1406, 1323, 1257, $1103 \mathrm{~cm}^{-1}$; MS: $\mathrm{m} / z$ $266.1\left[(\mathrm{M}+1)^{+}, 100 \%\right]$. Anal. Calcd for $\mathrm{C}_{11} \mathrm{H}_{11} \mathrm{BrN}_{2} \mathrm{O}: \mathrm{C}, 49.46 ; \mathrm{H}, 4.15 ; \mathrm{N}$, 10.49. Found: C, 49.63; H, 4.36; N, 10.06.

4-Bromo-5-methyl-1H-pyrazol-3-ol (2l) White solid; mp 192$194^{\circ} \mathrm{C}$; yield 85\%; ${ }^{1} \mathrm{H}$ NMR: $\delta 2.09$ (s, 3H, CH); IR: 2060-3270, 1587, 1253, 1172, $1053 \mathrm{~cm}^{-1}$; MS: $\mathrm{m} / z 176.9659\left[(\mathrm{M}+1)^{+}, 100 \%\right)$. Anal. Calcd for $\mathrm{C}_{4} \mathrm{H}_{5} \mathrm{BrN}_{2} \mathrm{O}: \mathrm{C}, 27.14 ; \mathrm{H}, 2.85 ; \mathrm{N}, 15.83$. Found: C, 27.26; H, 2.96; N, 15.66.

\section{Synthesis of 4-bromo-1-(4-bromophenyl)pyrazol- 3-ones 3b,j}

A mixture of $\mathbf{1 b}, \mathbf{j}$ (0.01 mol), $\mathrm{CCl}_{4}$ (10 mL) and hydrobromic acid (40\%, $0.05 \mathrm{~mol}$ ) was stirred, heated under reflux and treated dropwise with an aqueous solution of $\mathrm{H}_{2} \mathrm{O}_{2}(30 \%, 0.08 \mathrm{~mol})$ for $3 \mathrm{~h}$. After concentration under reduced pressure, the residue was chromatographed on silica gel eluting with ethyl acetate/petroleum ether (10:1) to give $\mathbf{3 b} \mathbf{b}$.

4-Bromo-1-(4-bromophenyl)-1H-pyrazole-3-ol (3b) Yellow solid; mp 199-201 ${ }^{\circ} \mathrm{C}$; yield 50\%; ${ }^{1} \mathrm{H}$ NMR: $\delta 11.17(\mathrm{~s}, 1 \mathrm{H}, \mathrm{OH}), 8.62(\mathrm{~s}, 1 \mathrm{H}, \mathrm{CH})$, 7.69 (m, 4H, Ar); IR: 3458, 2962, 1624, 1556, 1494, 1390, 1302, 1209, $1048 \mathrm{~cm}^{-1}$; MS: $\mathrm{m} / z 318.9\left[(\mathrm{M}+1)^{+}, 100 \%\right]$. 
4-Bromo-5-methyl-1-(4-bromophenyl)-1H-pyrazol-3-ol (3j) White solid; mp $243-245^{\circ} \mathrm{C}$; yield $58 \%$; ${ }^{1} \mathrm{H}$ NMR: $\delta 10.82(\mathrm{~s}, 1 \mathrm{H}$, $\mathrm{OH}$ ), 7.68 (d, $J=9 \mathrm{~Hz}, 2 \mathrm{H}, \mathrm{Ar}$ ), 7.45 (d, $J=9 \mathrm{~Hz}, 2 \mathrm{H}, \mathrm{Ar}$ ), 2.28 (s, 3H, $\mathrm{CH}_{3}$ ); IR: 3446, 2971, 1628, 1547, 1510, 1395, 1322, 1261, $1108 \mathrm{~cm}^{-1}$; MS: $\mathrm{m} / z 330.3\left[(\mathrm{M}+1)^{+}, 100 \%\right.$ ]. Anal. Calcd for $\mathrm{C}_{10} \mathrm{H}_{8} \mathrm{Br}_{2} \mathrm{~N}_{2} \mathrm{O}: \mathrm{C}, 36.18 ; \mathrm{H}$, 2.43; N, 8.44. Found: C, 36.33; H, 2.61; N, 8.28.

1-(4-Chlorophenyl)-1H-pyrazol-3-ol (4) White solid; mp 189$191^{\circ} \mathrm{C}$; ${ }^{1} \mathrm{H}$ NMR: $\delta 10.32$ (s, $\left.1 \mathrm{H}, \mathrm{OH}\right), 8.24$ (d, $\left.J=3 \mathrm{~Hz}, 1 \mathrm{H}, \mathrm{CH}\right), 7.70$ (d, $J=9 \mathrm{~Hz}, 2 \mathrm{H}, \mathrm{Ar}), 7.48$ (d, $J=9 \mathrm{~Hz}, 2 \mathrm{H}, \mathrm{Ar}), 5.84$ (d, $J=3 \mathrm{~Hz}, 1 \mathrm{H}, \mathrm{CH})$; IR: 3438, 2976, 1630, 1545, 1489, 1382, 1245, 1057, 945, $757 \mathrm{~cm}^{-1}$; MS: $\mathrm{m} / z$ $195.7\left[(\mathrm{M}+1)^{+}, 100 \%\right]$.

Acknowledgment: This project is supported by the Priority Academic Program Development of the Jiangsu higher education institutions (1107047002).

\section{References}

[1] Rapposelli, S.; Lapucci, A.; Minutolo, F.; Orlandini, E.; Ortore, G.; Pinza, M.; Balsamo, A. Synthesis and COX-2 inhibitory properties of $\mathrm{N}$-phenyl and $\mathrm{N}$-benzyl-substituted amides of 2-(4-methylsulfonylphenyl)cyclopent-1-ene-1-carboxylic acid and of their pyrazole, thiophene and isoxazole analogs. Farmaco 2004, 59, 25-31.

[2] Cottineau, B.; Toto, P.; Marot, C.; Pipaud, A.; Chenault, J. Synthesis and hypoglycemic evaluation of substituted pyrazole-4carboxylic acids. Bioorg. Med. Chem. Lett. 2002, 12, 2105-2108.

[3] Al-Omran, F.; El-Khair, A. A. Synthesis of polyfunctionally substituted heteroaromatic compounds via benzotriazolyl chalcones with antimicrobial and antifungal activities. J. Heterocycl. Chem. 2004, 41, 327-333.

[4] Sechi, M.; Sannia, L.; Carta, F.; Palomba, M.; Dallocchio, R.; Dessì, A.; Derudas, M.; Zawahir, Z.; Neamati, N. Design of novel bioisosteres of $\beta$-diketo acid inhibitors of HIV-1 integrase. Antiviral Chem. Chemother. 2005, 16, 41-61.

[5] Morita, H.; Harada, K.; Okamoto, Y.; Takagi, K. Ring transformation of 3-halo-4-methoxycoumarins into pyrazoles with hydrazines. J. Heterocycl. Chem. 1999, 36, 767-770.

[6] Li, Y.; Liu, Y. Y.; Chen, N. Q.; Lü, K. Z.; Xiong, X. H.; Li, J. One-pot regioselective synthesis of novel oximino ester-containing 1-aryl-4-chloro-3-oxypyrazoles as potential fungicides. Helv. Chim. Acta 2014, 97, 1269-1282.

[7] Arbačiauskienė, E.; Vilkauskaitė, G.; Eller, G. A.; Holzer, W.; Šačkus, A. Pd-catalyzed cross-coupling reactions of halogenated 1-phenylpyrazol-3-ols and related triflates. Tetrahedron 2009, 65, 7817-7824.

[8] Arbačiauskienė, E.; Martynaitis, V.; Krikštolaitytè, S.; Holzer, W.; Šačkus, A. Synthesis of 3-substituted 1-phenyl-1H-pyrazole4-carbaldehydes and the corresponding ethanones by Pdcatalysed cross-coupling reactions. Arkivoc 2011, 11, 1-21.

[9] Nedzelskytė, E.; Martynaitis, V.; Šačkus, A.; Eller, G. A.; Holzer, W. Synthesis of mono-and dibromo-derivatives of 1-phenylpyrazol-3-ol. Molbank 2007, 3, M551.

[10] Jørgensen, L.; Nielsen, B.; Pickering, D. S.; Kristensen, A. S.; Frydenvang, K.; Madsen, U.; Clausen, R. P. Analogues of 3-hydroxyisoxazole-containing glutamate receptor ligands based on the 3-hydroxypyrazole-moiety: design, synthesis and pharmacological characterization. Neurochem. Res. 2014, 39, 1895-1905.

[11] Arbačiauskienė, E.; Kazlauskas, K.; Miasojedovas, A.; Juršènas, S.; Jankauskas, V.; Holzer, W.; Getautis, V.; Šačkus, A. Multifunctional polyconjugated molecules with carbazolyl and pyrazolyl moieties for optoelectronic applications. Synth. Met. 2010, 160, 490-498.

[12] Arbačiauskienė, E.; Kazlauskas, K.; Miasojedovas, A.; Juršènas, S.; Jankauskas, V.; Holzer, W.; Getautis, V.; Šačkus, A. Pyrazolyl-substituted polyconjugated molecules for optoelectronic applications. Dyes Pigments 2010, 85, 79-85.

[13] Chande, M. S.; Bhandari, J. D.; Joshi, V. R. Investigation on the reaction of 4-anilino-5-mercapto-s-triazoles with pyrazolines and barbituric Acids. Indian J. Chem. B 1993, 32B, 1218-1228.

[14] El-Saraf, G. A.; El-Sayed, A. M.; El-Saghier, A. M. One-pot PTC synthesis of polyfused pyrazoles. Heteroat. Chem. 2003, 14, 211-217.

[15] Vogel, A. Vogel's Practical Organic Chemistry; Longman: London, 1978.

[16] Ahmed, S. A.; Awad, I. M.; Abdel-Wahab, A. M. A. A highly efficient photochemical bromination as a new method for preparation of mono, bis and fused pyrazole derivatives. Photochem. Photobiol. Sci. 2002, 1, 84-86.

[17] Huang, Y. Y.; Lin, H. C.; Cheng, K. M.; Su, W. N.; Sung, K. C.; Lin, T. P.; Huang, J. J.; Lin, S. K.; Wong, F. F. Efficient di-bromination of 5 -pyrazolones and 5 -hydroxypyrazoles by $\mathrm{N}$-bromobenzamide. Tetrahedron 2009, 65, 9592-9597.

[18] Cheng, K. M.; Wu, J. B.; Lin, H. C.; Huang, J. J.; Huang, Y. Y.; Lin, S. K.; Lin, T. P.; Wong, F. F. Dibromination of 5-pyrazolones and 5-hydroxypyrazoles via dibromoisocyanuric acid. J. Heterocycl. Chem. 2010, 47, 1153-1156.

[19] Frigola, J.; Colombo, A.; Pares, J.; Martinez, L.; Sagarra, R.; Roser, R. Synthesis, structure and inhibitory effects on cyclooxygenase, lipoxygenase, thromboxane synthetase and platelet aggregation of 3-amino-4, 5-dihydro-1H-pyrazole derivatives. Eur. J. Med. Chem. 1989, 24, 435-445.

[20] Li, Y.; Liu, R.; Yan, Z.; Zhang, X.; Zhu, H. Synthesis, crystal structure and fungicidal activities of new type oxazolidinonebased strobilurin analogues. Bull. Korean Chem. Soc. 2010, 31, 3341-3347.

[21] Liu, Y.; Li, Y.; Chen, N.; Xiong, X.; Yu, L.; Jing, C.; Zheng, Q.; Du, K.; Jiang, H. Synthesis, crystal structure, and fungicidal activity of novel 1-aryl-3-oxypyrazoles containing a Z-configuration methyl 2-(methoxyimino) acetate moiety. J. Chem. Res. 2014, 38, 520-523.

[22] Liu, Y.; Shi, H.; Li, Y.; Zhu, H. Synthesis, crystal structure, and fungicidal activity of novel 1,5 -diaryl-1H-pyrazol-3-oxyacetate derivatives. J. Heterocycl. Chem. 2010, 47, 897-902.

[23] Mercader, J. V.; Agulló, C.; Abad-Somovilla, A.; Abad-Fuentes, A. Synthesis of site-heterologous haptens for high-affinity antipyraclostrobin antibody generation. Org. Biomol. Chem. 2011, 9, 1443-1453.

[24] Saikia, I.; Borah, A. J.; Phukan, P. Use of bromine and bromoorganic compounds in organic synthesis. Chem. Rev. 2016, 116, 6837-7042. 
[25] Samanta, S.; Pappula, V.; Dinda, M.; Adimurthy, S. Transition metal-free oxidative esterification of benzylic alcohols in aqueous medium. Org. Biomol. Chem. 2014, 12, 9453-9456.

[26] Ghaffarzadeh, M.; Bolourtchian, M.; Tabar-Heydar, K.; Daryaei, I.; Mohsenzadeh, $\mathrm{F}$. $\mathrm{H}_{2} \mathrm{O}_{2}-\mathrm{HBr}$ : a metal-free and organic solventfree reagent system for the synthesis of arylaldehydes from methylarenes. J. Chem. Sci. 2009, 121, 177-182.

[27] Khan, A. T.; Parvin, T.; Choudhury, L. H.; Ghosh, S. A simple synthetic protocol for oxidation of alkyl-arenes into ketones using a combination of $\mathrm{HBr}-\mathrm{H}_{2} \mathrm{O}_{2}$. Tetrahedron Lett. 2007, 48, 2271-2274.

[28] O'Brien, D.; Gates Jr, J. Some reactions of 3-hydroxy-1-phenylpyrazole. J. Org. Chem. 1966, 31, 1538-1542.

Supplemental Material: The online version of this article offers supplementary material (https://doi.org/10.1515/hc-2018-0046). 